\title{
Produção de mudas de alface em substrato alternativo com adubação
}

\author{
Luiz Antonio Augusto Gomes ${ }^{1}$; Artenisa C Rodrigues²; Leonardo S Collier ${ }^{3}$; Selma dos S Feitosa ${ }^{2}$ \\ ${ }^{1}$ UFLA-DAG, C. Postal 3037, 37200-000, Lavras-MG; ${ }^{2}$ UFPB, Progr. Pós-graduação em Agronomia, 58397-000 Areia-PB; ${ }^{3}$ UFT-CUG, \\ C. Postal 66,77402-970 Gurupi-TO; laagomes@ufla.br; artenisacerqueira@hotmail.com leonardo@uft.edu.br
}

\section{RESUMO}

Substratos alternativos, além de permitirem a produção de mudas de qualidade, reduzem os custos de produção. Neste trabalho avaliou-se a produção de mudas de alface (Lactuca sativa L.) utilizando misturas de casca de arroz carbonizada e húmus de minhoca com diferentes doses de SulPoMag ${ }^{\circledR}$. O trabalho foi desenvolvido nas instalações da HortiAgro Sementes Ltda, em Ijaci (MG). Utilizou-se o delineamento de blocos casualizados, com cinco tratamentos e quatro repetições, sendo os tratamentos 1: $70 \%$ de húmus de minhoca (HM) e $30 \%$ de casca de arroz carbonizada (CAC), adicionados de 12,2 g de fosfato de Arad (FA) por litro da mistura, e tratamentos 2, 3 e 4: com as mesmas quantidades de HM, CAC, e FA, adicionadas, respectivamente, de 2,0; 4,0 e 8,0 $\mathrm{g}_{\text {de SulPoMag}}{ }^{\circledR}$. Como testemunha utilizou-se o substrato comercial Bioplant ${ }^{\circledR}$. Avaliaram-se as características de massa fresca de parte aérea (MFPA) e raiz (MFRZ); massa seca de parte aérea (MSPA) e raiz (MSRZ); teor de matéria seca de parte aérea (TMSPA) e raiz (TMSRZ) e

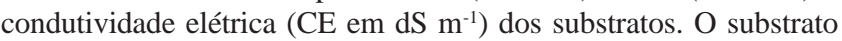
comercial e o tratamento 1 apresentaram maiores valores de MFPA e MSPA. Os outros tratamentos, independentemente das doses de SulPoMag ${ }^{\circledR}$, não diferiram entre si para MFPA. Para MSPA, o comportamento foi o mesmo, à exceção do tratamento 3 , que foi semelhante ao tratamento 1 e ao substrato comercial. $\mathrm{O}$ aumento das doses de SulPoMag ${ }^{\circledR}$ resultou em uma elevação da condutividade elétrica para todos os tratamentos de forma linear. A produção de mudas de alface semelhante àquela obtida com o substrato comercial é viável, utilizando-se matéria prima obtida na propriedade. A produção de MFPA foi afetada negativamente pela adição de SulPoMag ${ }^{\circledR}$ ao substrato.

Palavras-chave: Lactuca sativa L., substratos orgânicos, condutividade elétrica, casca de arroz carbonizada, húmus de minhoca.

\section{ABSTRACT \\ Production of lettuce seedlings using alternative substrates and fertilizer}

Alternative substrates, besides allowing the production of quality seedlings, reduce the production costs. In this work we evaluated the production of lettuce seedlings (Lactuca sativa L.) using mixtures of carbonized rice husk and earthworm humus with different doses of SulPoMag ${ }^{\circledR}$. The experimental design consisted of complete randomized blocks, with five treatments and four replicates. The evaluated mixtures consisted of treatment 1: $70 \%$ of earthworm humus (HM) and $30 \%$ of carbonized rice husk (CAC), with $12.2 \mathrm{~g}$ of Arad phosphate (FA) per each liter of mixture, and treatments 2, 3 and 4: formulated with the same quantities of HM, CAC and FA, added respectively with 2,0; 4,0 and 8,0 $\mathrm{g}$ of SulPoMag ${ }^{\circledR}$. The commercial substrate Bioplant ${ }^{\circledR}$ (SC) was used as control. The characteristics shoot fresh mass (MFPA), root fresh mass (MFRZ), shoot dry matter (MSPA), root dry matter (MSRZ), content of shoot dry matter (TMSPA), content of root dry matter (TMSRZ) and electrical conductivity ( $\mathrm{CE}$ in $\mathrm{dS} \mathrm{m}^{-1}$ ) were evaluated. The commercial substrate and the mixture 1 were superior to the others. Independent of the doses of SulPoMag ${ }^{\circledR}$, the mixtures did not present differences among them. The mean values of the treatments for MSPA were in the same tendency for MFPA, except for treatment 3, that presented production of MSPA similar to treatment 1 and the commercial substrate. Raising values of the electrical conductivity (CE) were observed in a linear way, in all mixtures, with increasing doses of SulPoMag ${ }^{\circledR}$. The production of lettuce seedlings is feasible similar to that obtained with the use of commercial substrate, using raw material found in the property. The production of MFPA was negatively affected by the addition of SulPoMag ${ }^{\circledR}$ in the substrate.

Keywords: Lactuca sativa L., organic substrates, electrical conductivity, carbonized rice husk, earthworms humus.

\section{(Recebido para publicação em 4 de dezembro de 2006; aceito em 6 de junho de 2008)}

\begin{abstract}
A alface (Lactuca sativa L.) está entre as hortaliças folhosas mais consumidas no mundo. Desenvolve-se melhor em regiões de clima ameno, ou em épocas do ano em que a temperatura permaneça na faixa de 15 a $20^{\circ} \mathrm{C}$ (Cásseres, 1980).

A base da horticultura moderna é a produção de mudas de alta qualidade (Scarpare Filho, 1994). Para produção e desenvolvimento de mudas em canteiros, casa-de-vegetação e recipientes limitados é comum modificar o solo ou mesmo criar substratos artificiais, usando materiais como turfa, composto cur-
\end{abstract}

tido, húmus de minhoca e casca de arroz. Esses substratos têm diferentes propriedades químicas e necessitam de monitoramento analítico prévio às correções e adubações (Ferreira et al., 1993). Buscando mostrar a aptidão de substratos na produção de mudas de hortaliças, Carrijo et al. (2002) observaram que houve uma leve superioridade do substrato à base de fibra de coco em termos absolutos na produção comercial de tomate, em relação a outros seis substratos utilizados.

Leal et al. (2007), utilizaram palhada de Crotalaria juncea L. e capim Napier
(Pennisetum purpureum Schum.) como substratos na produção de mudas de alface, beterraba e tomate, e mostraram que os substratos produzidos com misturas de $66 \%$ de crotalária e $33 \%$ de capim Napier foram os que mais se destacaram para todas as espécies utilizadas. Para o desenvolvimento de mudas de tomate, Fernandes et al. (2006) utilizaram substratos compostos por areia, bagaço de cana-de-açúcar e casca de amendoim, e observaram que as mudas com substratos que receberam bagaço de cana-de-açúcar e casca de amendoim apresentaram melhor desenvolvimento, 
podendo ser utilizados na composição de substratos à base de areia, para o cultivo do tomateiro.

Trani et al. (2007) trabalhando com uma cultivar de alface e três substratos comerciais, avaliaram as características químicas e físicas dos mesmos e concluíram que dentre os substratos avaliados, Plantmax HT $^{\circledR}$ demonstrou ser mais eficiente para produção de mudas de melhor qualidade. Em outro trabalho, também com alface, avaliando substratos comerciais, Trani et al. (2004), verificaram que o melhor desenvolvimento das mudas ocorreu em substrato Plantmax $\mathrm{HT}^{\circledR}$. O efeito de substratos formulados na propriedade como alternativa ao substrato comercial foi trabalhado por Medeiros et al. (2001) na produção de alface, constatando uma superioridade dos substratos húmus de minhoca + casca de arroz natural e húmus de minhoca + casca de arroz carbonizada em relação às demais misturas utilizadas para todas as características avaliadas.

O sistema de produção de mudas em bandejas de isopor começou a ser utilizado no Brasil a partir de 1984 (Minami, 1995), com vantagens, principalmente na produção de mudas mais uniformes. $\mathrm{Na}$ obtenção de maior número de mudas por unidade de área e melhor controle fitossanitário, resultando em mudas de melhor qualidade, os substratos (solos, substratos novos ou reutilizados), necessitam ser devidamente caracterizados para que se façam as correções e adubações necessárias. O manuseio e a utilização de misturas requerem cuidados especiais. Quatro problemas gerais podem ser considerados: acidez excessiva, excesso ou deficiência de nutrientes e salinidade, sendo que esta interfere diretamente na condutividade elétrica do substrato, podendo prejudicar ou até mesmo impedir o desenvolvimento das mudas (Bunt, 1976).

Visando alternativa para se produzir mudas de qualidade, a partir de matéria prima de fácil obtenção pelo agricultor associada a adubos orgânicos, o presente trabalho teve como objetivo avaliar a produção de mudas de alface utilizando mistura de casca de arroz carbonizada e húmus de minhoca, com adição de doses de SulPoMag ${ }^{\circledR}$.

\section{MATERIAL E MÉTODOS}

O experimento foi realizado na Estação Experimental da HortiAgro Sementes Ltda., em Ijaci (MG). O clima da região, conforme classificação de Köppen é Cwb, temperado úmido com inverno seco. As médias de temperaturas máxima e mínima ocorrem em fevereiro e julho, respectivamente, sendo a temperatura média de $19,3^{\circ} \mathrm{C}$. A precipitação média anual é de $1.411 \mathrm{~mm}$ e a umidade relativa do ar de 77,7\% (Brasil, 1992; Vianello, 1991).

Utilizou-se a cv. de alface Elba, de folhas consistentes, crespas e soltas, não formando cabeça. As mudas foram produzidas em bandejas de isopor de 200 células.

O delineamento experimental foi de blocos casualizados, com cinco tratamentos e quatro repetições. Tratamento 1 : $70 \%$ de húmus de minhoca (HM), 30\% de casca de arroz carbonizada (CAC) mais 12,20 g de fosfato de Arad (FA) por litro da mistura; Tratamento 2: $70 \%$ de húmus de minhoca (HM), 30\% de casca de arroz carbonizada (CAC) mais 12,20 g de fosfato de Arad (FA) e 2,00 $\mathrm{g}$ de SulPoMag ${ }^{\circledR}$ por litro da mistura; Tratamento 3: 70\% de húmus de minhoca (HM), 30\% de casca de arroz carbonizada (CAC) mais 12,20 $\mathrm{g}$ de fosfato de Arad (FA) e 4,00 g de SulPoMag ${ }^{\circledR}$ por litro da mistura; Tratamento 4: $70 \%$ de húmus de minhoca (HM), 30\% de casca de arroz carbonizada (CAC) mais 12,20 $\mathrm{g}$ de fosfato de Arad (FA) e 8,00 g de SulPoMag ${ }^{\circledR}$ por litro da mistura; Tratamento 5: testemunha, utilizandose o substrato comercial Bioplant ${ }^{\circledR}$.

$\mathrm{O}$ fosfato natural reativo (ARAD) foi adicionado às misturas com o objetivo de adicionar fósforo aos substratos. O SulPoMag ${ }^{\circledR}$ é uma rocha natural granular, que apresenta em sua composição $22 \%$ de $\mathrm{K}_{2} \mathrm{O}, 11 \%$ de $\mathrm{Mg}$ e $22 \%$ de $\mathrm{S}$, possuindo $99,7 \%$ de solubilidade.

As avaliações foram feitas no sentido de caracterizar cada substrato, no que diz respeito à sua condutividade elétrica e capacidade para formação e desenvolvimento de parte aérea e de raízes das mudas.

Para determinação da condutividade elétrica (CE) do substrato foram coletadas amostras compostas pelo substrato de 10 células de cada tratamento, em todas as repetições, em quatro épocas $(0 ; 7 ; 14$ e 21 dias após a semeadura). As amostras referentes a cada tratamento tiveram sua condutividade elétrica medida em $\mathrm{dS} \mathrm{m}^{-1}$.

As avaliações quanto à formação e desenvolvimento das mudas foram realizadas aos 21 dias após a semeadura em 10 plantas de cada parcela (uma fileira). MFPA (massa fresca de parte aérea): as plantas de cada tratamento foram seccionadas à altura do colo, separando-se a parte aérea da raiz. Em seguida obteve-se a massa da parte aérea das mesmas em gramas; MFRZ (massa fresca de raiz): as raízes de cada planta foram lavadas, retirando-se qualquer resíduo aderente às mesmas; em seguida foram mantidas à temperatura ambiente à sombra, para eliminar o excesso de água, sendo pesadas em balança de precisão; MSPA (massa seca de parte aérea): após a obtenção da MFPA, as plantas foram levadas para estufa a $65^{\circ} \mathrm{C}$, onde permaneceram por 24 horas, até atingirem o ponto de secagem; Em seguida foram pesadas em balança de precisão. A massa seca média por planta foi obtida dividindo-se a massa total obtida $\left(\mathrm{g}_{\text {planta }}{ }^{-1}\right)$, pelo número de plantas da parcela; MSRZ (massa seca de raiz): $\mathrm{O}$ mesmo procedimento foi feito para as raízes, obtendo-se os resultados em g planta ${ }^{-1}$; TMSPA (teor de matéria seca da parte aérea): expresso em percentagem, foi obtido pela relação entre a MSPA e a MFPA, multiplicando-se por 100; TMSRZ (teor de matéria seca de raiz): da mesma forma, o teor de matéria seca da raiz foi obtido pela divisão entre a MSRZ e a MFRZ, tendo os resultados expressos em percentagem.

As análises estatísticas foram realizadas utilizando-se o Software Sisvar para Windows, versão 4.3 (Ferreira, 2000), sendo efetuado teste de médias (Scott \& Knott, 1974) para as características com efeitos significativos.

Realizou-se análise de regressão para determinar as equações que melhor representassem as alterações ocorridas tanto na massa fresca da parte aérea (MFPA) quanto na condutividade elétrica (CE) dos substratos, em função das diferentes doses de SulPoMag ${ }^{\circledR}$ utiliza- 
das. Como a condutividade elétrica variou, dependendo da época de avaliação, utilizou-se para a análise de regressão a média da condutividade elétrica das quatro avaliações, para cada tratamento. Não foi realizada curva de regressão para o tratamento onde se utilizou o substrato comercial Bioplant ${ }^{\circledR}$, já que o mesmo não foi adicionado de SulPoMag ${ }^{\circledR}$. Devido à maior suscetibilidade de variações durante as manipulações do material radicular, as discussões serão concentradas em relação aos resultados referentes à massa fresca e seca da parte aérea.

\section{RESULTADOS E DISCUSSÃO}

Houve diferenças significativas para as características massa fresca da parte aérea (MFPA), massa seca de parte aérea (MSPA) e massa seca de raiz (MSRZ). Ao se comparar as médias para a característica massa fresca da parte aérea (MFPA), verifica-se que o substrato comercial Bioplant ${ }^{\circledR}$, juntamente com o Tratamento 1 (Tabela 1), substrato obtido à base da casca de arroz, húmus de minhoca e fosfato natural reativo (ARAD), sem adição de SulPoMag $^{\circledR}$ foram superiores aos demais, os quais não diferiram estatisticamente entre si.

Quando se analisa a massa seca da parte aérea (MSPA), os valores médios para cada tratamento seguem a mesma tendência da massa fresca da parte aérea (MFPA), exceto o Tratamento 3, que apresentou valor estatisticamente igual ao Tratamento 1 e ao substrato comercial Bioplant ${ }^{\circledR}$ (Tabela 1). Esses resultados sugerem viabilidade na produção de mudas de alface utilizando substrato alternativo à base de casca de arroz carbonizada e húmus de minhoca, adicionado de fosfato de Arad (Mota et al., 2003; Lana et al., 2004).

A maior produção de massa fresca da parte aérea, nos tratamentos com Bioplant ${ }^{\circledR}$ (Tabela 1), provavelmente possa ser explicada pelas diferenças ocorridas em relação à condutividade elétrica de cada substrato. A alface é uma cultura que normalmente tolera condutividade elétrica em torno de $1,3 \mathrm{dS} \mathrm{m}^{-1}$, podendo ter seu desenvolvimento prejudicado em condições de CE mais elevada (Ribeiro et al., 1999).

Tabela 1. Valores médios em gramas para as características de massa fresca da parte aérea (MFPA) e massa seca da parte aérea (MSPA) de mudas de alface produzidas em diferentes tipos de substrato (average values in grams, for fresh matter (MFPA) and dry matter of aerial part from lettuce seedlings produced in different kinds of substrates). Ijaci, UFLA, 2004.

\begin{tabular}{lcc}
\hline Tratamentos & MFPA (g) & MSPA (g) \\
\hline 1 & $3,6702 \mathrm{a}$ & $0,2735 \mathrm{a}$ \\
2 & $2,7565 \mathrm{~b}$ & $0,2157 \mathrm{~b}$ \\
3 & $3,0390 \mathrm{~b}$ & $0,2587 \mathrm{a}$ \\
4 & $2,0175 \mathrm{~b}$ & $0,1730 \mathrm{~b}$ \\
\hline Bioplant 8 & $3,7423 \mathrm{a}$ & $0,2765 \mathrm{a}$ \\
\hline
\end{tabular}

Médias seguidas da mesma letra na coluna não diferem estatisticamente entre si pelo teste de Scott \& Knott, ao nível de 5\% de probabilidade. (average folowed by the same letter in columns do not differ statistically between each other using Scott-Knott test to 5\% probability).

Tabela 2. Valores médios de condutividade elétrica em dS m${ }^{-1}$ de cinco substratos utilizados na produção de mudas de alface, em quatro épocas de avaliação (average values of eletrical conductance in $\mathrm{dS} \mathrm{m} \mathrm{m}^{-1}$ of five different substrates utilized in lettuce seedlings, in four evaluation dates). Ijaci, UFLA, 2004.

\begin{tabular}{lcccc}
\hline \multirow{2}{*}{ Tratamentos } & $\mathbf{4}$ Épocas de avaliação (dias) \\
\cline { 2 - 5 } & $\mathbf{0}$ & $\mathbf{7}$ & $\mathbf{1 4}$ & $\mathbf{2 1}$ \\
\hline 1 & $6,09 \mathrm{aC}$ & $3,32 \mathrm{bD}$ & $2,99 \mathrm{cD}$ & $3,29 \mathrm{cD}$ \\
2 & $6,96 \mathrm{aB}$ & $4,49 \mathrm{bC}$ & $3,08 \mathrm{cD}$ & $3,59 \mathrm{cD}$ \\
3 & $7,77 \mathrm{aB}$ & $4,22 \mathrm{bC}$ & $2,79 \mathrm{cD}$ & $4,07 \mathrm{bC}$ \\
4 & $10,67 \mathrm{aA}$ & $5,19 \mathrm{bC}$ & $3,61 \mathrm{cD}$ & $3,96 \mathrm{cD}$ \\
5 & $4,57 \mathrm{cD}$ & $3,83 \mathrm{cD}$ & $3,01 \mathrm{cD}$ & $3,68 \mathrm{cD}$ \\
\hline
\end{tabular}

Médias seguidas pela mesma letra maiúscula (avaliação) e minúscula (tratamentos) não diferem estatisticamente entre si (Scott \& Knot, 1974) (averages followed by the same capital letters (avaliação) and small letters (treatments) do not differ statistically between each other) (Scott \& Knot, 1974).

Para verificar a influência do SulPoMag ${ }^{\circledR}$ na produção de massa fresca da parte aérea das plantas fez-se uma análise de regressão para esta característica em função das doses de SulPoMag ${ }^{\circledR}$ (Figura 2). Pode-se verificar que à medida que aumentou a dose de SulPoMag ${ }^{\circledR}$ houve uma redução linear na produção de massa fresca da parte aérea. A produção de MFPA reduziu pela adição de SulPoMag ${ }^{\circledR}$ ao substrato na proporção de 0,18 gramas de MFPA para cada dois gramas de SulPoMag ${ }^{\circledR}$ adicionados.

Para as características relacionadas aos substratos, em que se avaliou a condutividade elétrica em quatro épocas distintas, houve efeitos significativos entre os substratos, entre as épocas de avaliação e para a interação entre os dois fatores.

Ao se fazer o desdobramento da interação (Tabela 2) das diferentes misturas de substratos dentro das quatro épocas de avaliação, verifica-se que, na primeira avaliação (instalação do experimento), a condutividade elétrica variou entre os diferentes tipos de substrato. Ela foi inferior no substrato Bioplant ${ }^{\circledR}(4,57$ $\mathrm{dS} \mathrm{m}^{-1}$ ), que diferiu estatisticamente dos demais. O Tratamento 1 , cujo valor de $6,09 \mathrm{dS} \mathrm{m}^{-1}$ também foi estatisticamente diferente dos demais. Já os Tratamentos 2 e 3 não diferiram entre si e apresentaram valores de $6,96 \mathrm{dS} \mathrm{m}^{-1}$ e 7,77 $\mathrm{dS} \mathrm{m}{ }^{-1}$, respectivamente. Com valor significativamente superior, o Tratamento 4 apresentou condutividade elétrica nesta primeira avaliação de $10,67 \mathrm{dS} \mathrm{m} \mathrm{m}^{-1}$. Observa-se que, de maneira geral, a condutividade elétrica foi superior nas misturas que receberam maior dose de SulPoMag ${ }^{\circledR}$, embora a condutividade elétrica do substrato comercial não se alterou ao longo do experimento (Menezes Júnior et al., 2000, Trani et al., 2007).

No substrato Bioplant ${ }^{\circledR}$ não houve diferença estatística ao longo das quatro avaliações, sendo observado valor médio de $3,77 \mathrm{dS} \mathrm{m}^{-1}$. Para os demais 


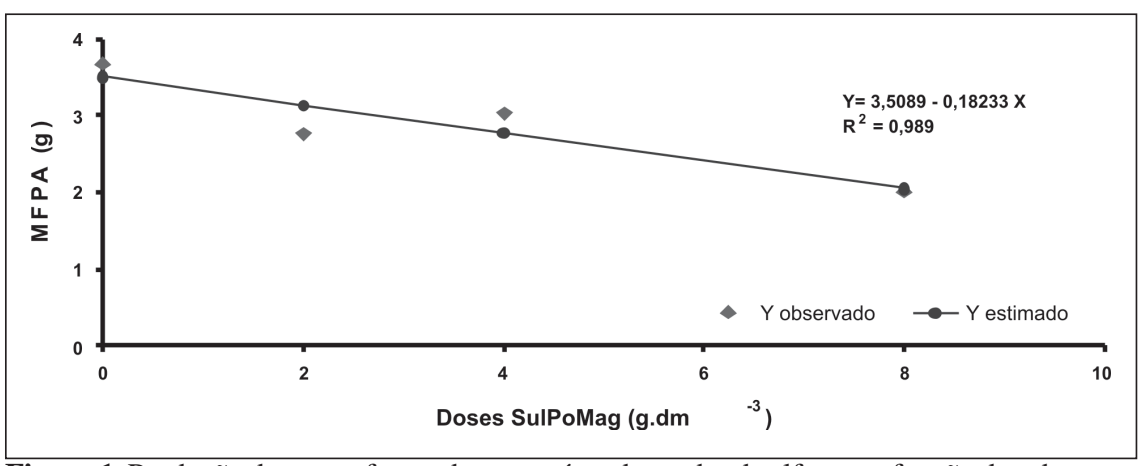

Figura 1. Produção de massa fresca da parte aérea de mudas de alface em função de substratos com diferentes doses de SulPoMag ${ }^{\circledR}$ (fresh matter production of lettuce seedlings according to substrates with different levels of SulPoMag ${ }^{\circledR}$ ). Ijaci, UFLA, 2004.

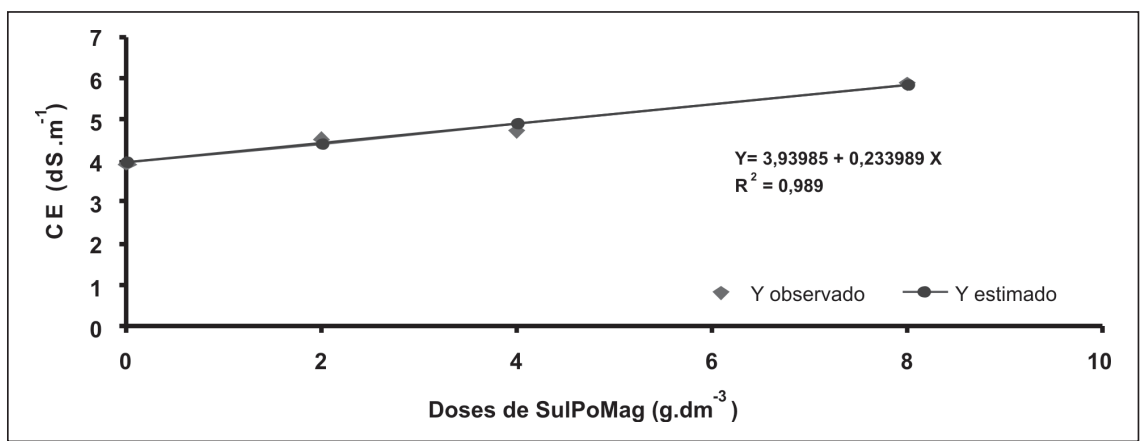

Figura 2. Condutividade elétrica média de substratos em função do aumento de dose de SulPoMag ${ }^{\circledR}$ (average electric conductance of substrates according to increasing level of SulPoMag ${ }^{\circledR}$ ). Ijaci, UFLA, 2004.

substratos houve uma redução significativa nos valores da condutividade elétrica a partir da segunda avaliação, aos sete dias. Neste caso o Tratamento 1, ao qual não foi adicionado o SulPoMag ${ }^{\circledR}$, apresentou valor de $3,32 \mathrm{dS} \mathrm{m}^{-1}$, igualando-se ao substrato Bioplant ${ }^{\circledR}$, com valor de $3,83 \mathrm{dS} \mathrm{m}^{-1}$. Os Tratamentos 2 , 3 e 4, aos quais foram adicionado o SulPoMag ${ }^{\circledR}$ em diferentes concentrações, não diferiram estatisticamente entre si (Tabela 2), porém apresentaram valores estatisticamente superiores de 4,49 dS m ${ }^{-1}, 4,22 \mathrm{dS} \mathrm{m}^{-1}$ e $5,19 \mathrm{dS} \mathrm{m}^{-1}$, respectivamente. Em hortaliças como o repolho, moderadamente sensível à salinidade, a redução de rendimento em relação à condutividade elétrica do extrato de saturação do solo (CEes) para os valores de 1,$8 ; 2,8 ; 4,4 ; 7,0$; e 12,0 $\mathrm{dS} \mathrm{\textrm {m } ^ { - 1 }}$ foi, respectivamente, de 0,25 , 50 e $100 \%$ de perdas (Doorenbos \& Kassam, 1994). Já a partir da terceira avaliação (14 dias após a instalação do experimento), a condutividade elétrica entre os substratos não diferiu estatisticamente entre si (Tabela 2).
Provavelmente, a redução da condutividade elétrica de cada substrato esteja relacionada ao efeito da irrigação, que promoveu lixiviação do excesso de sais. Assim, a planta pode ter absorvido parte desses nutrientes presentes em formas iônicas biodisponíveis no substrato.

Ao se avaliar a condutividade elétrica aos vinte e um dias (Tabela 2), momento que corresponde à época do transplantio das mudas e na qual também se avaliou a massa fresca da parte aérea (MFPA) das plantas, verifica-se que não houve diferença significativa entre os substratos, à exceção do Tratamento 4, que apresentou uma ligeira elevação da condutividade elétrica. O Tratamento 1 foi aquele no qual ocorreu uma redução mais acentuada da condutividade elétrica, já na avaliação feita aos sete dias (Tabela 2).

Apesar da condutividade elétrica ao final do experimento não diferir estatisticamente para os diferentes tipos de substrato, ao se fazer uma análise de regressão, verifica-se que ao contrário da produção de massa fresca da parte aérea (Figura 1), ocorreu um aumento linear nos valores da condutividade elétrica média (Figura 2), em função do aumento das doses de SulPoMag ${ }^{\circledR}$. A elevação da condutividade elétrica média, ocorrida pelo aumento das doses de SulPoMag ${ }^{\circledR}$, foi fator decisivo para a redução da massa fresca da parte aérea das plantas. Portanto, a utilização de SulPoMag ${ }^{\circledR}$ adicionado ao substrato alternativo contribuiu para a elevação da condutividade elétrica média do mesmo e reduziu as produções de MFPA e MSPA.

\section{AGRADECIMENTOS}

À UFLA, à HortiAgro Sementes Ltda. e aos colegas que colaboraram na execução do trabalho.

\section{REFERÊNCIAS}

BRASIL. 1992. Ministério da Agricultura e Reforma Agrária. Normais Climatológicas. 1961-1990. Brasília: MARA, 84p.

BUNT AC. 1976. Modern Potting Composts. Londres. George Allen \& Unwin. 277p.

CARRIJO OA; LIZ RS; MAKISHIMA N. 2002. Fibra da casca do coco verde como substrato agrícola. Horticultura Brasileira 20: 533-535.

CÁSSERES E. 1980. Producción de hortalizas. San José, Costa Rica: Instituto Interamericano de Ciências Agrícolas, 387p.

DOORENBOS J; KASSAM AH. 1994. Efeito da água no rendimento das culturas. Paraíba-PB: UFPB, 306p.

FERNANDES C; CORÁ JE; BRAZ LT. 2006. Desempenho de substratos no cultivo do tomateiro do grupo cereja. Horticultura Brasileira 24: 42-46.

FERREIRA DF. 2000. Manual do sistema Sisvar para análises estatísticas. Lavras: UFLA, 66p.

FERREIRA ME; CASTELLANE PD; CRUZ MCP. 1993. Nutrição e adubação de hortaliças. Piracicaba-SP, 487p.

LANA RMQ; ZANÃO JÚNIOR LA; LUZ JMQ; SILVA JC. 2004. Produção da alface em função do uso de diferentes fontes de fósforo em solo de Cerrado. Horticultura Brasileira 22: 525-528.

LEAL MAA; GUERRA JGM; PEIXOTO RTG; ALMEIDA DL. 2007. Utilização de compostos orgânicos como substratos na produção de mudas de hortaliças. Horticultura Brasileira 25: 392-395.

MEDEIROS LAM; MANFRON PA; MEDEIROS SLP; BONNECARRÈRE RAG. 2001. Crescimento e desenvolvimento da alface (Lactuca sativa $L$.) conduzida em estufa plástica com fertirrigação em substratos. Ciência Rural 31: 199-204.

MENEZES JÚNIOR FOG; FERNANDES HS; MAUCH CR; SILVA JB. 2000. Caracterização de diferentes substratos e seu desempenho na produção de mudas de alface em ambiente protegido. Horticultura Brasileira 18: 164-170. 
MINAMI K. Produção de Mudas de Alta Qualidade em Horticultura. São Paulo. TA. Editor. 1995. 128p.

MOTA JH; YURI JE; RESENDE GM; OLIVEIRA CM; SOUZA RJ; FREITAS SAC; RODRIGUES JÚNIOR JC. 2003. Produção de alface americana em função da aplicação de doses e fontes de fósforo. Horticultura Brasileira 21: 620-622.

RIBEIRO AC; GUIMARÃES PTG; ALVAREZ VH. 1999. (Eds.) Comissão de fertilidade do solo do Estado de Minas Gerais: Recomendações para o uso de corretivos e fertilizantes em Minas Gerais - $5^{a}$ Aproximação. Viçosa, MG.
SCARPARE FILHO JA. 1994. "Mudas de frutíferas de alta qualidade". In: MINAMI K; TESSARIOLI NETO J; PENTEADO SR; SCARPARE FILHO JA. Produção de mudas hortículas de alta qualidade, p.16-21. USP/ SEBRAE, Piracicaba.

SCOTT AJ; KNOTT M. 1974. Acuster analysis method for grouping means in the analysis of variance. Biometrics 30: 507-512.

TRANI PE; FELTRIN DM; POTT CA; SCHWINGEL M. 2007. Avaliação de substratos para produção de mudas de alface. Horticultura Brasileira 25: 256-260.
TRANI PE; NOVO MCSS; CAVALLARO JÚNIOR ML; TELLES LMG. 2004. Produção de mudas de alface em bandejas e substratos comerciais. Horticultura Brasileira 22: 290294.

VIANELLO LR. 1991. Meteorologia básica e aplicações. Viçosa: UFV. 